\title{
BERICHT DER VERBUNDZENTRALE DES ÖSTERREICHISCHEN BIBLIOTHEKENVERBUNDES ÜBER DEN ZEITRAUM MAI 2019 BIS APRIL 2020
}

\section{Redaktion: Wolfgang Hamedinger und Mathis Kronschläger, Text: Team der OBVSG}

Zusammenfassung: Aufbauend auf dem verbundinternen, jährlichen „Bericht der Verbundzentrale", wird die Arbeit des Österreichischen Bibliothekenverbundes und seiner Verbundzentrale vom Zeitraum Mai 2019 bis April 2020 einer breiten Öffentlichkeit im Überblick vorgestellt.

Schlagwörter: Österreichischer Bibliothekenverbund; OBV; Die Österreichische Bibliothekenverbund und Service GmbH; OBVSG; Verbundzentrale; Jahresbericht; Projekte; Dienstleistungen

\section{REPORT OF THE MAIN OFFICE OF THE AUSTRIAN LIBRARY NETWORK OVER THE PERIOD FROM MAY 2019 TO APRIL 2020}

Abstract: Drawing from the internal, annual report of the Austrian Library Network's main office, this article presents projects and services from May 2019 to April 2020 to the broader public.

Keywords: Austrian Library Network; OBV; Austrian Library Network and Services Ltd; OBVSG; main office; headquarter; annual report; projects; service

DOI: https://doi.org/10.31263/voebm.v73i2.3942

(c) Die Österreichische Bibliothekenverbund und Service GmbH (OBVSG)

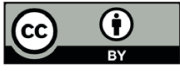

Dieses Werk ist - exkl. einzelner Logos und Abbildungen - lizenziert unter einer Creative-Commons-Lizenz Namensnennung 4.0 International 


\section{Inhaltsverzeichnis}

Vorbemerkung

1. Der Verbund und seine Verbundzentrale

1.1. Neues zu Verbundteilnehmern und Interessenten

2. Statistikwerte

3. Alma-Implementierung

3.1. Migrationen

3.2. Zentrales System (Netzwerkzone) und Parallelbetrieb

3.3. Kreiskonvertierung, Kreishybridisierung und Katalogisierung

3.4. Zentrale Services

4. Laufende Projekte

4.1. Digitale Langzeitarchivierung

4.2. MARC21-Titelimport/-export

4.3. Daten: Analysen / Korrekturen / Pflege / Web-Plattform

4.4. Weiterentwicklung des RDA-Toolkits (3R-Projekt)

5. Abgeschlossene Projekte

5.1. Digitaler Assistent

5.2. Infrastruktur

6. Verbundübergreifende Zusammenarbeit

7. Aktueller Stand von OBVSG-Diensten

7.1. Zeitschriftendatenbank (ZDB) als Fremddatenquelle/Normdatei

7.2. Gemeinsame Normdatei (GND)

7.3. SAP-Schnittstelle

7.4. eDOC: Repository und Kataloganreicherung

7.5. Primo

7.6. Bestandsdatenlieferung an die ZDB

7.7. EasyTool

7.8. MetaLib/SFX

7.9. E-Book-Datenversorgung

7.10. Bibliotheksstatistik

7.11. Adressdatenbank (ACC09) / OBVSG als ISIL-Agentur

7.12. Visual Library

7.13. URN(-Resolver)

7.14. Memo-Workflow

7.15. Statistikwerte aus den OBVSG-Diensten

8. Termine

9. Organisatorisches und Sonstiges

9.1. IGeLU

9.2. Geplante Novellierung OBVSG-Gesetz 


\section{Vorbemerkung}

Der vorliegende Bericht enthält keine vollständige Zusammenstellung aller laufenden Aktivitäten der OBVSG, sondern behandelt bemerkenswerte Entwicklungen seit dem Bericht für die Vollversammlung des Österreichischen Bibliothekenverbundes vom 16. Mai 2019.

Ursprünglich wurde das vorliegende Dokument als Bericht der Verbundzentrale für die Vollversammlung am 22. April 2020 geplant. Da dieser Termin aufgrund der COVID-19-Pandemie ohne konkreten Ersatztermin abgesagt werden musste, erscheint dieser Bericht nun in leicht abgewandelter Form. Der ursprünglich vorgesehene Berichtszeitraum blieb gleich, ebenso der dafür festgelegte Redaktionsschluss. Lediglich Termine und Veranstaltungen, die wegen der derzeitigen Situation abgesagt werden mussten, wurden korrigiert.

Die OBVSG hat auf die Corona-Pandemie schnell reagiert und binnen kürzester Zeit auf Heimarbeit umgestellt. Die dafür erforderliche technische Infrastruktur wie beispielsweise Laptops für Mitarbeiterinnen und Mitarbeiter war bereits im Kern vorhanden und konnte für den Einsatz von zuhause aus eingerichtet werden. Dienste wie Primo, Aleph-Hosting und Aleph-Sharing, Alma-ASP, SAP und Visual Library konnten auch in Krisenzeiten weiter fortgeführt und betreut werden, ebenso die Migrationsprojekte der Wave 7 und Wave 8.

Der weitgehende Verzicht auf persönliche Treffen im Arbeitskontext, zählt zu den Herausforderungen, die es in dieser Ausnahmesituation zu meistern gilt. Sowohl für die interne als auch die externe Kommunikation konnte dafür auf bestehende Strukturen wie beispielsweise Video- und Telefonkonferenzen, das OBVSG-Ticketsystem und nicht zuletzt auf den Schriftverkehr via Email aufgebaut werden. Vor allem für die interne Kommunikation wurden neue Lösungen geschaffen, die vielleicht auch über die Krisensituation hinaus erhalten bleiben und das Arbeitsleben nach der Rückkehr in den regulären Betrieb erleichtern werden.

\section{Der Verbund und seine Verbundzentrale}

Der Österreichische Bibliothekenverbund (OBV) ist der große Verbund der wissenschaftlichen und administrativen Bibliotheken Österreichs - darunter die Österreichische Nationalbibliothek, die Universitätsbibliotheken, die Pädagogischen Hochschulen, einzelne Ministerien, zahlreiche Fachhochschulen und weitere bedeutende Sammlungen. Der Verbund ist offen für die Teilnahme weiterer Einrichtungen. 
Die Österreichische Bibliothekenverbund und Service GmbH (OBVSG) agiert als die Dienstleistungs- und operative Leitungseinrichtung des OBV. Zu den Kernaufgaben der OBVSG als Verbundzentrale gehören unter anderem: der operative Betrieb des Verbundsystems, Systemadministration und Softwarepflege, Bereitstellung eines Systems zur Verbundkatalogisierung, Bereitstellung einer Recherche-Oberfläche mittels Suchmaschinentechnologie, Datenmanagement - insbesondere Konvertierungen, Prüfungen und Korrekturen, systembibliothekarischer Support, die zentrale Pflege der Fremd- und Normdatenpools und die Bereitstellung von Schnittstellen zu allen deutschen Verbünden und der Deutschen Nationalbibliothek.

\subsection{Neues zu Verbundteilnehmern und Interessenten}

Die aktuelle Liste aller Verbundteilnehmer findet sich unter: https://www. obvsg.at/bibliothekenverbund/verbundbibliotheken-liste/

\subsubsection{Neue Verbundteilnehmer}

Die Salzburg Museum GmbH ist mit 1. Juli 2019 dem Verbund beigetreten. Von einem BIBLIOTHECAplus System ausgehend werden rund 170.000 Datensätze in eine Alma Institutionszone im Rahmen der Wave 9 migriert werden.

\subsection{2. Änderungen}

Bedauerlicherweise ist die ÖFSE - Österreichische Forschungsstiftung für Internationale Entwicklung mit Ende 2019 aus dem Verbund ausgeschieden. 


\section{Statistikwerte}

Wesentliche Kennzahlen aus Alma:

\begin{tabular}{|l|c|c|}
\hline & 31.01 .2020 & 31.03 .2019 \\
\hline Titeldaten & 15.280 .603 & 14.137 .238 \\
\hline - mit AC-Nummern & 13.781 .659 & 13.556 .584 \\
\hline - aus der Community Zone & 1.498 .944 & 580.670 \\
\hline Zeitschriftenbestandsdaten & 745.810 & 734.389 \\
\hline Exemplardaten physisch & 28.965 .826 & 25.708 .134 \\
\hline Anzahl aktiver Portfolios & 6.697 .503 & 3.847 .655 \\
\hline Zeitschriftendatenbank ZDB & 421.264 & 416.851 \\
\hline
\end{tabular}

Wesentliche Kennzahlen aus Aleph:

\begin{tabular}{|l|c|c|c|}
\hline & 31.01 .2020 & 31.03 .2019 & 01.04 .2018 \\
\hline Deutsche Nationalbibliographie DNB & 7.393 .904 & 6.798 .456 & 6.509 .477 \\
\hline Casalini Libri & 408.440 & 387.900 & 366.353 \\
\hline Gemeinsame Normdatei GND & 16.086 .468 & 15.236 .986 & 14.750 .544 \\
\hline
\end{tabular}

\section{Alma-Implementierung}

\subsection{Migrationen}

\subsubsection{Projektorganisation und Allgemeines}

Weiterhin wird das Wave-Management zentral von der OBVSG durchgeführt. Die OBVSG übernimmt darüber hinaus, wo sinnvoll und notwendig und nach Maßgabe der verfügbaren Ressourcen, die Koordination zwischen Ex Libris und den Verbundteilnehmern und fungiert als zentraler Ansprechpartner für Fragen die Netzwerkzone betreffend.

Alle Erkenntnisse, die im Laufe der Migration der Waves gewonnen werden, werden im „Dossier“ festgehalten und bereitgestellt. Das Dossier und seine Ergänzungen werden laufend aktualisiert und ergänzt.

Sehr bewährt hat sich das sogenannte „Onboarding“, welches auch für alle weiteren Waves beibehalten wird. Dieses findet einige Monate vor dem eigentlichen Kick-off statt und soll die Teilnehmenden mit den Grundzügen des Projekts vertraut machen. Insbesondere die Einzelgespräche mit den Einrichtungen erlauben es beiden Seiten, sich schon zu einem frühen Zeitpunkt inhaltlich abzustimmen. 


\subsubsection{Aleph-Sharing-Einrichtungen / Sharingtag}

Am 27. Februar 2020 fand der Sharingtag in den Räumlichkeiten der OBVSG statt. Da durch das Fortschreiten der Waves immer mehr Bibliotheken mit Alma produktiv sind, wurde das Treffen auf „Sharingtag und Treffen der Alma-ASP-Bibliotheken" umbenannt. Im Mittelpunkt standen die Themen Collections, Benutzerverwaltung und Statistiken in Alma, Neues in Primo, der Digitale Assistent, Aufgaben der lokalen Redaktion u.v.m.

Die OBVSG führt weiterhin Einschulungen in die Module Katalogisierung (inkl. MARC-21), Erwerbung und Ausleihe durch. Schulungen zur Verwaltung von E-Ressourcen und Fernleihe wurden von Einrichtungen des Bibliothekenverbundes übernommen.

\subsubsection{Wave 5}

In der Wave 5 gingen am 4. September 2019 folgende Einrichtungen live mit Alma:

- Akademie der bildenden Künste Wien

- CAMPUS 02 Fachhochschule der Wirtschaft GmbH

- Fachhochschule Salzburg GmbH

- FH Campus Wien

- FHW Fachhochschul-Studiengänge Betriebs- und Forschungseinrichtungen der Wiener Wirtschaft $\mathrm{GmbH}$

- Oberösterreichische Landeskultureinrichtungen

- Paracelsus Medizinische Privatuniversität Salzburg - Privatstiftung

- Universität für Weiterbildung Krems (Donau-Universität)

Die Akademie der bildenden Künste Wien und die Oberösterreichischen Landeskultureinrichtungen übernahmen dabei mit eigenem Personal die systembibliothekarischen Aufgaben des Systemwechsels.

\subsubsection{Wave 6}

In der Wave 6 gingen am 13. Jänner 2020 folgende Einrichtungen live mit Alma:

- Österreichische Galerie Belvedere (Research Center - Bibliothek)

- Benediktinerstift Admont (Archiv und Bibliothek)

- Bibliotheken Wien (Wienbibliothek im Rathaus)

- Diözesanbibliothek Wien

- Jüdisches Museum der Stadt Wien 
- Lentos Kunstmuseum Linz

- Montanuniversität Leoben

- Technische Universität Graz

- Wiener Wiesenthal Institut für Holocaust-Studien

Als neuer Verbundteilnehmer erforderte die Migration des Belvedere von BIBLIOTHECAplus auf Alma, welche zur Gänze von der OBVSG durchgeführt wurde, eine andere Herangehensweise als bei den Aleph-Bibliotheken. Ausgehend vom Gesamtdatenabzug wurden die bibliographischen und exemplarspezifischen BIBLIOTHECAplus-Daten auf Grundlage einer ausgefeilten Konkordanz in das passende MARC-21 Format gebracht, in die korrekte Datenstruktur umgewandelt und danach über die Alma-API in die neue Alma-Institutionszone eingespielt. Neben der mit erheblichem Aufwand erarbeiteten Konkordanz waren Programme für die Datenkonversion und das parallele Laden in Alma zu erstellen, wobei sich die inzwischen gewonnenen Erfahrungen mit Alma und die langjährigen Kontakte mit Ex Libris als sehr hilfreich erwiesen. Für die Migration der Benutzerdaten und Entlehnungen konnten generische Alma-Mittel genutzt werden.

Neu in Wave 6 war auch, dass die OBVSG für die vollständige Migration von vier Aleph-Sharing-Einrichtungen selbst zuständig war: diese waren Stift Admont, Lentos, Jüdisches Museum und Wiener Wiesenthal Institut für Holocaust-Studien. Alle vier hatten ausschließlich bibliographische Daten, Holdings und Items zu migrieren. Als Methode wurde die bewährte MAB-MARC-Konvertierung und der Extraktionsmechanismus aus Aleph gewählt. Die so extrahierten METS-Container wurden anschließend programmtechnisch aufbereitet, in Ladefiles für Alma umgewandelt und über die Alma-API eingespielt.

Die im Rahmen der Wave 6 entwickelten Werkzeuge werden in adaptierter Form auch in den nachfolgenden Waves und bei etwaigen späteren Migrationen aus Fremdsystemen wiederverwendet. Insbesondere werden sie in Wave 7 bei der Migration der Anton-Bruckner-Privatuniversität, als auch in Wave 9 beim Salzburg Museum zum Einsatz kommen.

\subsubsection{Wave 7}

In der Wave 7 stehen seit dem Onboarding vom 23. Oktober 2019 und dem Kick-off vom 12. Februar 2020 folgende Einrichtungen am Start:

- Anton Bruckner Privatuniversität

- Diözese Linz (mit der Privaten Pädagogischen-Hochschule und der Diözesan- und Universitätsbibliothek) 
- Fachhochschule des BFI Wien GmbH

- Fachhochschule Kärnten

- FH JOANNEUM Gesellschaft mbH

- FH OÖ Studienbetriebs GmbH (FH Oberösterreich)

- Universität für künstlerische und industrielle Gestaltung Linz

- Universität für Musik und darstellende Kunst Wien

- Universität Mozarteum Salzburg

Die Universität für künstlerische und industrielle Gestaltung Linz, die Universität für Musik und darstellende Kunst Wien und die Universität Mozarteum Salzburg werden die Migration mit eigenem systembibliothekarischem Personal durchführen, die anderen Einrichtungen werden systembibliothekarisch von der OBVSG betreut.

Als neuer Verbundteilnehmer wird die Anton Bruckner Privatuniversität von einem DABIS-System kommend migrieren. Analog zur Migration aus anderen Nicht-Aleph-Systemen wird die Erstellung der Konkordanz, die programmtechnische Datenkonversion und das Laden der Daten über die Alma-API durch die OBVSG durchgeführt.

Der geplante Betriebsaufnahmetermin der Wave 7 ist der 1. September 2020.

\subsubsection{Wave 8 und 9}

Mit dem Onboarding der Wave 8 am 2. April 2020 (per Videokonferenz) begann bereits die Endphase des Migrationszyklus. In dieser Wave migrieren die letzten Aleph-Sharing Einrichtungen, nämlich: Graphische Sammlung Albertina, Bundesministerium für europäische und internationale Angelegenheiten, Diözesanbibliothek Salzburg im Archiv der Erzdiözese Salzburg, Diözese Gurk-Klagenfurt, Diözese St. Pölten, Fachhochschule Technikum Wien, MODUL University Vienna, New Design University, Salzburger Landesarchiv, Sigmund Freud Privatstiftung sowie Stiftsbibliothek Heiligenkreuz.

Wie bereits in Wave 6 führt auch in Wave 8 die OBVSG die Migration für folgende Einrichtungen selbständig durch: Graphische Sammlung Albertina, Diözesanbibliothek Salzburg im Archiv der Erzdiözese Salzburg sowie Bibliothek der Sigmund Freud Privatstiftung.

Nach der Produktionsaufnahme der Wave 8 im Jänner 2021 werden die Aleph-Sharing-Systeme (SH1, SH2 und SH3) nach einer kurzen Auslaufphase im Frühjahr 2021 abgeschaltet.

Der Migrationszyklus des Österreichischen Bibliothekenverbundes endet mit den Lokalsystemen der Bibliotheken der Wave 9: Fachhoch- 
schule Kufstein Tirol Bildungs GmbH (Alephino), Pädagogische Hochschule Burgenland (Alephino), Parlamentsbibliothek, Universität für angewandte Kunst Wien und voraussichtlich Bundesministerium für Inneres / Sicherheitsakademie (Alephino). Das Salzburg Museum wird von einem BIBLIOTHECAplus-System migrieren. Gleiches plant die Universität für Musik und darstellende Kunst Graz.

Mit der geplanten Produktionsaufnahme der Wave 9 im September 2021 samt Nacharbeiten endet das große Umstiegsprojekt im OBV.

\subsection{Zentrales System (Netzwerkzone) und Parallelbetrieb}

Der Parallelbetrieb zwischen Alma und Aleph läuft im Großen und Ganzen störungsfrei.

Die Alma Netzwerkzone (sowie die Institutionszonen) kämpfen immer wieder mit Performanceproblemen insbesondere das Publishing nach Primo betreffend. Teilweise kam es über Tage hinweg zu keiner Datenversorgung nach Primo. Die OBVSG bemüht sich in diesen Fällen Druck auf Ex Libris auf verschiedenen Ebenen auszuüben und erreicht damit immerhin, dass Hotfixes sofort und nicht erst mit dem nächsten Release eingespielt werden. Das grundlegende Problem der mangelnden Performance besteht aber weiterhin.

\subsection{Kreiskonvertierung, Kreishybridisierung und Katalogisierung}

Der MAB-MARC-Konverter inklusive der sogenannten „Kreishybridisierung" bildet nach wie vor als Kreiskonverter für die Datenkonversion der bibliografischen Daten zwischen der Alma-Netzwerkzone (Primärdatenbank) und dem zentralen Aleph-System (Aleph-Bridge) das entscheidende Werkzeug für die Datenintegrität während des Parallelbetriebs von Alma und Aleph.

Das OBVSG-Ticketsystem ist weiterhin Basis sowohl für Korrekturen und Optimierungen des MAB-MARC-Konverters als auch für Korrekturen und Optimierungen von Normalisierungsregeln und weiteren Set-up-Einstellungen im Bereich der bibliografischen Daten in Alma.

Durch die Katalogisierungspraxis in Alma und umfangreiche Datenanalysen sind laufend Konverteranpassungen und Datenkorrekturen notwendig. Das MARC21-Format wird zunehmend in seinem breiten Spektrum in der Katalogisierung angewendet. Folgende Bereiche waren im Berichtszeitraum und sind weiterhin von Anpassungen im Kreiskonverter besonders betroffen: 
- Kodierungen in fixen Feldern

- Detailänderungen in bestehenden Feldern

- Änderungen in der Katalogisierungspraxis vor allem im Bereich der Aufsatzkatalogisierung und der Hybridaufnahmen

Darüber hinaus müssen auch folgende Komponenten weiterentwickelt und gepflegt werden, um die einheitliche Vorgangsweise im Verbund sicherzustellen:

- Implementierung neuer und Optimierung bestehender Normalisierungsregeln in Alma für

- Datenanpassungen beim Abspeichern von Datensätzen

- Datenkorrekturen

- Import und Importprofile für externe Ressourcen bzw. E-BookDatenpakete

- Satz erweitern und Schreibvorlagen für Nachlasskatalogisierung, P2E und E2P (gedruckt $\leftrightarrow$ elektronisch)

- Merge-Routinen für Anreicherung aus externen Ressourcen

- Pflege der zentralen Katalogisierungsschablonen

- Pflege des MARC21-Extension-Packs inkl. Controlled Vocabulary

Der Arbeitsaufwand dafür ist erheblich und wird sich für den weiteren Parallelbetrieb auch kaum reduzieren lassen.

\subsection{Zentrale Services}

\subsubsection{Memos und Erinnerungen}

Die koordinierte Verarbeitung von Löschungen und anderen Aktionen, bei denen eine verbundweite Katalogkoordination nötig ist, wurde um die Verarbeitung des Typs „ZDB-OK“ erweitert.

Der neue Typ ist konsortial aufgesetzt, sodass alle Verbundteilnehmer den Workflow hierfür nutzen können.

Verarbeitungsstatistik von 06.04.2019 bis 21.02.2020:

\begin{tabular}{|l|c|c|}
\hline & Löschmemos & ZDB-Ok \\
\hline erfolgreich verarbeitet & 17.162 & 2.772 \\
\hline zurückgewiesen & 5.560 & 80 \\
\hline
\end{tabular}




\section{Laufende Projekte}

\subsection{Digitale Langzeitarchivierung}

Die OBVSG und die Österreichische Nationalbibliothek befinden sich derzeit in einem gemeinsamen Ausschreibungsverfahren für ein (konsortialfähiges) System zur digitalen Langzeitarchivierung. Die Auftragsbekanntmachung erfolgte am 27. September 2019 auf der TED-Plattform ${ }^{1}$.

Der Projektplan sah zunächst eine Zuschlagsentscheidung bzw. den Vertragsabschluss im Juli/August 2020 vor, die Implementierung des Systems hätte dann im Herbst 2020 starten sollen. Auf Grund des durch die COVID-19-Maßnahmen pausierten Vergabeverfahrens werden sich diese Meilensteine jedoch um zumindest zwei Monate verzögern.

\subsection{MARC21-Titelimport/-export}

Folgende DNB-Export-Releases wurden fristgerecht für Alma und für Aleph in Produktion genommen:

- DNB-Export-Release 2019.02 (Juni 2019)

- DNB-Export-Release 2019.03 (Oktober 2019)

- DNB-Export-Release 2020.01 (Februar 2020)

Im Jahr 2020 sind noch die DNB-Export-Releases 2020.02 (Juni 2020) und 2020.03 (September 2020) zu implementieren.

Für die GND-Lieferungen bzw. der Arbeit mit der Online-Schnittstelle war im Berichtszeitraum das Release 2020.01 (Version 1.9, gültig ab 16. Oktober 2018) zu implementieren.

Die Anreicherungen mit Sacherschließungselementen und Links (Inhaltsverzeichnisse der DNB) wurden rückwirkend seit Produktivnahme der Alma-Netzwerkzone nachgezogen. Anschließend wurde ein tägliches Update via OAl-Harvesting eingerichtet.

Damit erfolgen die Aktualisierungen von Datensätzen aus den Quelldaten nunmehr auch in Alma wie bisher in Aleph, allerdings deutlich häufiger, mit weniger Aufwand durch die Verwendung von Standardmechanismen und ohne Notwendigkeit einer Pflege lokaler Spiegel der Quelldatenbanken. 


\subsection{Daten: Analysen / Korrekturen / Pflege / Web-Plattform}

\subsubsection{Entwicklungen / Aktueller Stand}

Auf Basis der Gespräche sowie der Erkenntnisse im Bereich des MABMARC-Konverters wurden folgende Maßnahmen getroffen bzw. umgesetzt:

- Erweiterungen für die Konkordanz lokaler Felder im Dokument „Zentrale und lokale MARC21-Felder im OBV“

- Datenstatistiken für migrierende Institutionen

- Reports zu 98X-Feldern auf Netzwerkzonenebene

- Fehlerauswertungen (Datenmonitor) zu Memos (Aleph) und Erinnerungen (Alma)

\subsection{Weiterentwicklung des RDA Toolkits (3R-Projekt)}

Durch die Einführung des IFLA Library Reference Models (LRM) wurde der RDA-Regelwerkstext sowie das derzeit verwendete RDA Toolkit einer grundsätzlichen Umgestaltung unterzogen. Dies hat nicht nur Auswirkungen für die deutschsprachigen Anwendungsrichtlinien (DACHAWR) sondern auf die Erschließungspraxis insgesamt. Hierzu hat die Fachgruppe Erschließung (Standardisierungsausschuss) einen ersten Evaluations(zwischen)bericht vorgelegt: Mit dem neuen RDA-Regelwerkstext liegt ein abstraktes Rahmenregelwerk vor, das lexikonartig und sehr komplex aufgebaut ist - um für die praktische Anwendung eine logische Struktur herzustellen und das Verständnis des Textes zu ermöglichen, sind zusätzliche Erläuterungen und Workflows notwendig, die erheblich über den Umfang der bisherigen DACH-AWR hinausgehen. Als Gesamtfazit wurde festgehalten, dass das derzeit vorliegende Beta-Toolkit mit dem neuen Regelwerkstext nicht praxistauglich ist.

Diese tiefgreifenden Veränderungen, die international und auch im deutschsprachigen Raum sowohl hinsichtlich ihrer Inhalte als auch hinsichtlich der Präsentation dieser bereits massiv als praxisuntauglich kritisiert wurden, haben den Standardisierungsausschuss dazu veranlasst, die Fachgruppe Erschließung mit der Überprüfung und einer Aufwandsschätzung für die Entwicklung von Alternativen - soweit möglich im Rahmen des RDA Toolkits - zu beauftragen. Eine Aufgabe des RDA Toolkits an sich könnte der weiterhin bestechenden Idee einer einheitlichen weltweiten Grundlage für die Erschließung von Materialien einen möglicherweise zerstörerischen Schlag versetzen. 
Anhand ausgewählter praktischer, verbreiteter Anwendungsfälle sollen alltagstaugliche, formatunabhängige Anwendungsrichtlinien basierend auf den neuen RDA-Regelwerkstexten ausgearbeitet und die praktische Umsetzung der Arbeitsergebnisse in ihren Auswirkungen beschrieben werden. Außerdem wurde die Fachgruppe Erschließung gebeten, Modelle für eine alltagstaugliche und möglichst ressourcenschonende Dokumentation dieser Anwendungsrichtlinien im Zusammenspiel mit dem RDA Toolkit zu präzisieren. Diese Aufträge an die Fachgruppe Erschließung sollen auch dazu dienen, den Aufwand für die Umsetzung des 3R-DACH-Projekts sowie die Auswirkungen für die praktische Erschließungsarbeit einzuschätzen.

Näheres siehe unter folgendem Link: https://wiki.dnb.de/display/ RDAINFO/3R-DACH-Projekt

Da keine signifikanten Kostensteigerungen erfolgen, wird die OBVSG den österreichischen Lizenzgebührenanteil für das bisherige RDA Toolkit auch im Jahr 2020 ohne Weiterverrechnung übernehmen.

\section{Abgeschlossene Projekte}

\subsection{Digitaler Assistent}

Der Digitale Assistent (DA-3) ist ein von der Schweizer Softwarefirma Eurospider und der Universitätsbibliothek Stuttgart entwickeltes webbasiertes Vorschlagstool zur Unterstützung der Sacherschließerinnen und Sacherschließer in ihrer jeweiligen Verbundumgebung. Diese bisher hauptsächlich im PICA-Umfeld eingesetzte Software wurde für die direkte Integration mit Alma durch die Firma Eurospider erweitert und durch die OBVSG an Alma angebunden; die Bearbeitenden sehen - im Gegensatz zur derzeitigen Einbindung in PICA - unmittelbar alle Ergebnisse in Alma, aber auch etwaige Fehlermeldungen und können während des Arbeitsablaufs ohne Verzögerung entsprechend reagieren. Es handelt sich um die erste Implementierung bei der die Daten unmittelbar nach Eingabe in den Katalog übernommen werden.

In dem Integrationsprojekt wurde die Datenübernahme aus dem Digitalen Assistenten und die fachliche Vorverarbeitung der Datensätze nach der im OBV vereinbarten Katalogisierungspraxis implementiert. Für den Erfolg war die Kooperation mit Expertinnen und Experten des Verbunds wesentlich.

Damit können nun über viele Möglichkeiten im Verbundkatalog des Österreichischen Bibliothekenverbundes und in den Katalogen der deutschen Verbünde nach Vorschlägen für die Erschließung der eigenen Res- 
source gesucht und geeignete Ergebnisse sofort und bequem in die eigenen Datensätze übernommen werden.

Die von den Nutzerinnen und Nutzern erfassten Daten werden von Eurospider an eine Schnittstelle der OBVSG zur direkten Integration in den Verbundkatalog geliefert. Die hinter den verwendeten Zugangskennungen liegenden persönlichen Daten sind nur der OBVSG bekannt, sodass auch keine datenschutzrechtlichen Probleme auftreten können.

Der Dienst ist seit 20. Jänner 2020 im OBV verfügbar. Am 18. und 19. Februar 2020 fanden Schulungen statt, um eine gute Basis für die künftige Verwendung in den Institutionen zu legen. Das Umsetzungsprojekt ist damit abgeschlossen und der Dienst befindet sich im Regelbetrieb.

Der Dienst wird je genannter Person bestellt und abgerechnet.

\subsection{Infrastruktur}

Die kontinuierliche Erneuerung der Komponenten der produktiven Infrastruktur der OBVSG dient nicht zuletzt der Einhaltung der jeweils aktuellen technischen Standards.

Bei beiden mit Ende des Vorjahres erneuerten Firewall-Appliances wurden im Laufe des Berichtszeitraums fortgeschrittene Schutzmaßnahmen wie Malware-Protection und Intrusion-Detection mit Unterstützung unseres Supportdienstleisters schrittweise verschärft, erprobt und in Betrieb genommen.

Die redundante Netzwerkinfrastruktur am Produktionsstandort bei Nessus (6 Stk. HPE Procurve E6600) wurde mit Ende 2019 erneuert und durch aktuelle Geräte des Typs Cisco Nexus 3048T ersetzt. Mit Beginn 2020 erfolgte die Übernahme in den Produktivbetrieb.

Im Laufe des Juni 2019 wurden die bisher verwendeten Schulungs-Clients (HPE Thinclients mit separatem Bildschirm und separater Tastatur und Maus) durch 25 neue HPE-Notebooks mit 17"-Bildschirm (1920×1080 px) ersetzt, sodass nun auch die Ansprüche von Benutzerschnittstellen aktueller Softwaresysteme erfüllt werden. Nicht zuletzt werden damit Auf- und Abbau der Geräte im Schulungsraum nennenswert vereinfacht und beschleunigt ${ }^{2}$.

\section{Verbundübergreifende Zusammenarbeit}

Neben der routinenmäßigen Zusammenarbeit liegen die Schwerpunkte weiterhin bei Alma. Im Berichtszeitraum wurden nach den nötigen Ent- 
scheidungen die Umstellungsarbeiten in der Schweiz, bei der erstmalig eine Gesamtlösung für das ganze Land angestrebt wird (Swiss Library Service Platform) und in Nordrhein-Westfalen begonnen. Beide Großprojekte profitieren von den österreichischen Vorarbeiten und umgekehrt wird auch Österreich von den zusätzlichen Entwicklungen in den beiden Projekten profitieren. Schlussendlich wird eine deutlich stärkere deutschsprachige Alma-Anwendergemeinde entstehen, die eine ähnliche Systemarchitektur verwendet.

\section{Aktueller Stand von OBVSG-Diensten}

\subsection{Zeitschriftendatenbank (ZDB) als Fremddatenquelle/Normdatei}

Die Zeitschriftendatenbank (ZDB) ist die zentrale bibliografische Datenbank für Titel- und Besitznachweise fortlaufender Sammelwerke in Deutschland und Österreich. Im OBV wird die Zeitschriftendatenbank als Normdatei geführt, die ZDB-Koordination ist daher bemüht weitere Einrichtungen für die aktive ZDB-Eingabe zu gewinnen.

Beitritte im Berichtszeitraum:

- Fachhochschule St. Pölten

- Akademie der bildenden Künste Wien

Beide Einrichtungen haben im Jänner 2020 den WebCat-Produktionszugang von der OBVSG erhalten.

Für das im Rahmen des Verbundtags 2019 präsentierte Katalogisierungshandbuch (Bereich fortlaufende Ressourcen) konnten seither weitere Inhalte erstellt werden. Die Einarbeitung und Aktualisierung der Texte wurde größtenteils von der ZDB-Koordination übernommen.

Im Bereich der Altdatenkorrektur wurden für 42.725 ÖZDB-Zeitschriftentitel der Verbunddatenbak maschinelle Änderungen bei der ZDB-ID durchgeführt. Die Redakteurin der ÖNB und die ZDB-Koordination waren vorab für die Datenanalyse verantwortlich. Das Metadatenteam der OBVSG hat am 21. Oktober 2019 alle ÖZDB-Nummern ohne Entsprechung in der ZDB gelöscht, bzw. durch „ZDB-NEU-9999-99-99“ ersetzt.

Datenpflege und damit auch Korrekturmeldungen von Zeitschriftentiteln in der Verbunddatenbank sind für die Arbeit der ZDB-Redaktion von hoher Relevanz. Noch nicht in der ZDB vorhandene Titel werden im OBV mit „ZDB-NEU-JJJJ-MM-TT“ gekennzeichnet. Bei über 15.000 Zeitschrif- 
ten- und Zeitungstiteln fehlt der im Workflow vorgesehene Eintrag. Die ZDB-Redaktion wird sich beim nächsten Redaktionstreffen mit dieser Thematik beschäftigen.

Jeweils am Monatsende werden von der OBVSG alle Datensätze (Zeitschriften/Zeitungen) maschinell auf Stufe 90 gehoben, die über die OAISchnittstelle der ZDB aktualisiert wurden und eine Katalogisierungsstufe kleiner als 90 haben. Die Anhebung der Katalogisierungsstufe wird von der Koordinationsstelle durchgeführt.

Für die gezielte Aktualisierung eines Zeitschriftentitels mit dem aktuellen Stand aus der ZDB ist in Alma eine Erinnerung, in Aleph ein Memo „ZDB-OK“ für die Verarbeitung zu setzen. Am 5. Februar 2020 hat die OBVSG die Produktion für Erinnerungen/Memos „ZDB-OK“ live geschaltet. Unter Nutzung der SRU-Schnittstelle der DNB konnten 2.678 Erinnerungen/Memos des initialen Laufs abgearbeitet werden. Im Katalogisierungshandbuch wurde von der Koordinationsstelle die Seite Erinnerung/ Memo „ZDB-OK“ aktualisiert. Projektbeteiligung: Entwicklungsteam, Metadatenteam und ZDB-Koordination der OBVSG.

Die in der WebEx der Zeitschriftenexpertinnen und -experten (4. Juni 2019) angesprochene fehlende Primo-Anzeige von Datensätzen mit Beziehung Vorgänger/Nachfolger (MARC 780 bzw. 785) konnte, in Absprache mit der ZDB-Koordination, vom Primo-Team der OBVSG noch im Juni 2019 erfolgreich implementiert werden.

Am Österreichischen Bibliothekartag in Graz hat erstmals eine ZDB-Infoveranstaltung stattgefunden. Es bestand die Möglichkeit für Einzelgespräche mit den Redakteuren der OBVSG, UB Graz und der Redakteurin der ÖNB.

Mit dem Systemwechsel der Kunstuniversität Linz werden 2020 alle Einrichtungen des ZDB-Redaktionskollektivs im OBV mit Alma produktiv sein.

\subsection{Gemeinsame Normdatei (GND)}

Die Gemeinsame Normdatei (GND) ist eine an der Deutschen Nationalbibliothek vorgehaltene Normdatei für Personen, Körperschaften, Veranstaltungen, Geografika, Sachschlagwörter und Werktitel. Sie wird von der Deutschen Nationalbibliothek in Kooperation mit den deutschsprachigen Bibliotheksverbünden und weiteren bibliothekarischen Einrichtungen geführt. Normdaten erleichtern die Katalogisierung, bieten eindeutige Sucheinstiege und die Möglichkeit der Vernetzung unterschiedlicher Informationsressourcen. Die OBVSG stellt mit dem Softwareanbieter die GND den Verbundmitgliedern sowohl in Aleph als auch in Alma in Form zweier Spiegeldatenbanken zur Verfügung. Sie beteiligt sich zudem über den Ko- 
ordinator der GND-Level-1-Redaktion des OBV in diversen Bereichen aktiv an der GND-Datenpflege.

Hauptthema für die GND-Level-1-Redaktion war im Berichtszeitraum erneut der Systemwechsel von Aleph auf Alma. Diesbezüglich können in allen Teilbereichen große Fortschritte vermeldet werden.

Im Teilbereich Normdatenpflege wurden die zuletzt noch vorhandenen Probleme im dritten Quartal 2019 von Ex Libris behoben, sodass im vierten Quartal die mehrmals verschobenen Umstiegsschulungen stattfinden konnten. In Vorbereitung auf diese wurden diverse Dokumente wie Schulungsunterlagen, Erfassungsleitfäden und -hilfen neu erstellt bzw. an die Gegebenheiten in Alma angepasst.

An drei Terminen im November wurden Schulungen für Multiplikatoren und Multiplikatorinnen abgehalten und insgesamt knapp 70 Personen aus den Bibliotheken bis zur Wave 5 auf rein technischer Ebene in der Normdatenpflege in Alma unterwiesen. Diese werden das erworbene Wissen in weiterer Folge an alle betroffenen Kolleginnen und Kollegen an der jeweils eigenen Institution weitergeben. Ende Februar 2020 fand die GND-inAlma-Schulung für die Wave-6-Bibliotheken unter den selben Rahmenbedingungen statt, sodass mit März 2020 bereits mehr als die Hälfte der Verbundbibliotheken die Normdatenpflege in Alma durchführen können. Die Schulungen für die folgenden Waves sind jeweils kurz nach dem Start der produktiven Phase geplant.

In Bezug auf den Alma-GND-Spiegel wurden diverse Änderungen im Format, dem kontrollierten Vokabular etc. vom OBV zunächst in den internationalen Gremien mit erarbeitet und dann gemeinsam mit Ex Libris im System umgesetzt. Nicht zuletzt wurden auch die nötigen Vorbereitungen für die geplante Löschung der Namenssätze aus der GND getroffen. Darüber hinaus wurden auch Maßnahmen erarbeitet, um den Alma-GND-Spiegel bzgl. Datenbasis auf den neuesten Stand zu bringen. Die Umsetzung der letztgenannten Punkte wird im Sommer 2020 erfolgen.

Im Teilbereich der automatisierten Korrektur der Titeldaten (Aufgabenliste Normdatenverwaltung - ALNV) wurde eine vom OBV seit langem geforderte Normalisierung in Bezug auf die unterschiedliche Darstellung von Sonderzeichen in Titel- und GND-Datensätzen implementiert, wodurch ein effizientes Monitoring dieser Prozesse in greifbare Nähe rückt.

Während der Teilbereich Nutzung der GND zum Erschließen von Ressourcen (F3-Funktion) bereits seit Anfang 2019 problemlos funktioniert, wurde im Teilbereich Retrieval durch eine Verbesserung bzgl. Granularität der veröffentlichten Daten eine langjährige Forderung des OBV umgesetzt. Diese ist unmittelbar vor allem für Musik-Bibliotheken von Bedeutung 
(38X-Felder), kann potentiell aber positive Auswirkungen auf alle Kataloge haben.

Des Weiteren wurde in punkto Retrieval mit dem OBVSG-Primo-Team und dem Team Standardisierung der OBVSG weiter an einer verbesserten Indexierung bzw. Darstellung der GND-Daten in den Suchmaschinen gearbeitet. Seit April 2020 sind diese Neuerungen im Verbund-Primo verfügbar und ermöglichen eine explizite Normdatenrecherche.

Abseits von Alma wurden mit der Planung der nächsten Runde der Regelwerksschulungen (COVID-19-bedingt derzeit geplant für Herbst 2020, statt ursprünglich April/Mai 2020), der Implementierung des GND-Teiles des OBV-Katalogisierungshandbuches und der Einführung eines GNDNewsletters wichtige Maßnahmen zur Sicherung der Datenqualität getroffen.

Im GND-Ausschuss lag das Hauptaugenmerk der Tätigkeit, neben den oben erwähnten operativen Anpassungen, auf den strategischen Themen der Öffnung der GND für Kultureinrichtungen, aber auch Verlage und der Vorbereitung auf das 3R-Projekt.

Letztlich hat die OBVSG vergangenes Jahr Kontakt zu anderen, derzeitigen und künftigen, GND-Anwendern aufgenommen und wird versuchen, gemeinsam mit anderen Institutionen bzw. Verbünden eine Alma-GNDAnwender-Gruppe zu bilden, um hinkünftig in Alma notwendige Adaptionen mit gemeinsamer, und dadurch hoffentlich gewichtigerer Stimme, einfordern zu können. Ursprünglich war dies im Rahmen der DACHELA im Juni 2020 in Wien geplant, da diese jedoch aufgrund COVID-19 abgesagt wurde, steht noch kein Zeitpunkt für ein Treffen fest.

\subsection{SAP-Schnittstelle}

Die SAP-Schnittstelle für Aleph lief in der Berichtsperiode ohne Probleme und wird mit Umstellung des letzten Anwenders im Sommer 2020 obsolet.

Die SAP-Schnittstelle für Alma läuft weiterhin in der Version 0.9 und soll aufVersion 1.0 gebracht werden, sobald es die Ressourcensituation erlaubt. Diese Version wird neben der Ergänzung kleinerer Funktionalitäten alle 10-15 Minuten auf das Vorhandensein neuer Daten aus Alma prüfen und damit nicht nur einmal täglich die von Alma ausgegebenen Daten verarbeiten.

Weiters wurde auf Grund eines Anwenderauftrags das Übertragungsformat erweitert. Die erforderlichen Tests waren bis Ende des Berichtszeitraums nicht abgeschlossen. Da die Erweiterung parametrisierbar ist, hat die Änderung keine Auswirkung auf die anderen Schnittstellennutzer. 


\section{4. eDOC: Repository und Kataloganreicherung}

eDOC ist ein Service der OBVSG zur Anreicherung des Verbundkataloges mit elektronischen Dokumenten. Die Anwendung unterstützt Objekttypen wie Inhaltsverzeichnisse, Abstracts, Rezensionen, Umschlagbilder und Klappentexte sowie Volltextdokumente und Bilder. Diese können mit Primo über Metadaten und Inhalte durchsucht werden.

Die Gesamtzahl der Objekte im eDOC-Repository beträgt mit Stand 5. Februar 2020 1.157.606 Objekte, davon 768.990 Inhaltsverzeichnisse, 45.697 Abstracts und 62.433 Volltexte.

Folgende Einrichtungen nehmen an eDOC (Variante eDOClight) teil:

- Universität für Bodenkultur

- CAMPUS 02 Fachhochschule der Wirtschaft

- FHW Fachhochschul-Studiengänge

- Parlament

- Akademie der bildenden Künste Wien

- Universität für Musik und darstellende Kunst Wien

- Universität Linz

- Wienbibliothek im Rathaus

- Universität Salzburg

\subsection{Primo}

\subsubsection{Allgemeines}

Primo macht alle Ressourcen einer Institution unter einer gemeinsamen Oberfläche suchbar. Dadurch werden die Daten der diversen Einzelsysteme im Hintergrund aufbereitet, sodass sie unter einheitlicher Oberfläche und mit aktueller Suchmaschinentechnologie recherchiert und dargestellt werden können. Neben dem Auffınden wird auch der Zugang zur benötigten Information gewährleistet (Entlehn-Informationen, Bestellung, Vormerkung, Download etc.).

Technische und organisatorische Grundlage aller Primo-Sucheinstiege ist eine konsortiale Primo-Instanz, angesiedelt an der OBVSG.

Im Berichtszeitraum wurde von Primo Release Februar 2019 bis zu Primo Release Februar 2020 gewechselt. Insgesamt wurden vier neue Service Packs eingespielt.

Neuerungen, die durch die Service Packs für das Primo realisiert wurden, sind unter anderem: 
- Citation Trails - Zitiermöglichkeiten

- Erweiterte Konfigurationsmöglichkeiten von Alert-E-Mails

Weitere neue Funktionalitäten, die unabhängig von den Service Packs realisiert wurden:

- Verlinkung von Vorgänger und Nachfolger: Diese Verlinkung ist bei Zeitschriften sehr hilfreich, weil es bei diesen immer wieder zu Namensänderungen kommt. Vor der Umsetzung wurde Rücksprache mit der ZDB-Redaktion gehalten, um die Funktion korrekt abzubilden.

- Collection Discovery: Die Verbundsuchmaschine wurde um den Bereich „Sammlungen“ (https://search.obvsg.at/primo-explore/collec tionDiscovery?vid=OBV\&lang=de_DE) erweitert. Hier werden Nachlässe und Handschriften ab sofort in den Teilsammlungen Bestand, Werk, Korrespondenz, Lebensdokument und Sammelstück übersichtlich dargestellt und können gezielt durchsucht werden.

- Einbindung von Thumbnails: Dank einer Kooperation mit der ÖNB ist es gelungen, in der Verbundsuchmaschine weitere 450.000 Thumbnails aus den Digitalisierungsprojekten $A B O$ und $A N N O$ in der Kurzanzeige einzubinden.

- E-Ressourcen im Verbund: Auf Wunsch des Primo-Fachbeirats wurde ein neuer Suchbereich in der Verbundsuchmaschine erstellt. In diesem sind E-Ressourcen, die in Alma in der Community Zone von den einzelnen Bibliotheken aktiviert wurden, durchsuchbar.

\subsubsection{Neues User Interface (NUI)}

Nach dem erfolgreichen Pilotprojekt für den Umstieg auf das Neue User Interface (NUI) im Vorjahr standen nun gleich zwei Umstellungen für das Jahr 2019 an. Einerseits hat sich der Verbund für Bildung und Kultur (VBK) dazu entschlossen, auf das NUI umzusteigen, andererseits sollte auch die Verbundsuchmaschine im neuen Design erscheinen. Für beide Umstellungen wurden Projekte angelegt und eine Fertigstellung für September 2019 angestrebt. Dies war nicht nur notwendig, um aktuellen Nutzererwartungen gerecht zu werden, sondern auch, weil Ex Libris im Sommer 2019 den Support für das alte User Interface eingestellt hat.

\section{$V B K$}

Im Zuge der Umstellung des VBK wurden zusätzliche Features programmiert. Im Vordergrund stand die Umsetzung der Selbstanmeldung, die 
seit der Umstellung auf Alma ein großer Wunsch des VBK war. Außerdem finden sich die unterschiedlichen Views der verschiedenen Pädagogischen Hochschulen nun unter einer einheitlichen Oberfläche wieder. Beibehalten wurden die unterschiedlichen Sucheinstiege sowie der Suchbereich für die Semesterliteratur. Am 6. September 2019 kam es zum Relaunch des NUI für den VBK.

\section{Verbundsuchmaschine}

Gleichzeitig mit dem Projekt VBK wurde auch die Umstellung der Verbundsuchmaschine realisiert. Dafür wurde ein Grafiker engagiert, der das Layout der Verbundsuchmaschine gestalten sollte. Da dieser bereits für das Layout der alten Suchoberfläche verantwortlich zeichnete, verlief die Kommunikation problemlos. Das Layout wurde mit besonderem Augenmerk auf die Aktualisierung der Medienicons erarbeitet und in Folge technisch umgesetzt. Zusätzlich zu den Suchseiten wurde auch die Hilfeseite komplett überarbeitet und für mobile Endgeräte optimiert. Nach einer längeren Testphase ging die Verbundsuchmaschine mit dem neuen Design pünktlich zum Österreichischen Bibliothekartag am 16. September 2019 online.

\section{Nachlassverzeichnis}

Ein weiteres Projekt im Hinblick auf das NUI war die Aktualisierung und Neugestaltung des „Verzeichnisses der künstlerischen, wissenschaftlichen und kulturpolitischen Nachlässe in Österreich" (NLV). Dieser Katalog war noch auf den Aleph-OPAC aufgesetzt und sollte in Zusammenarbeit mit der Österreichischen Nationalbibliothek auf Primo NUI umgestellt werden.

Bei diesem Projekt gab es einige Herausforderungen: Der Katalog ist dreisprachig, viele der bisherigen Suchfunktionen ließen sich nicht einfach in Primo einbinden und die Daten sollten mittels Harvesting aus der AlmaNetzwerkzone nach Primo geladen werden. Diese Art der Datenversorgung wurde seit der Umstellung auf Alma zum ersten Mal umgesetzt. So können gezielt nur jene Daten nach Primo geladen werden, die auch wirklich relevant sind. Am 9. Oktober 2019 ging das neue Nachlassverzeichnis in Betrieb.

\section{Weitere Umstiege}

Zusätzlich sind im Berichtszeitraum folgende Bibliotheken auf das NUI umgestiegen: 
- Universität Wien

- Universität Innsbruck

- Universität Salzburg

- Universität Graz

\subsubsection{Entwicklung der Teilnehmer}

Im Berichtszeitraum haben folgende Bibliotheken den produktiven Betrieb aufgenommen:

- Technische Universität Graz (Jänner 2020)

\subsubsection{Organisatorisches}

\section{Supportmeeting mit Ex Libris}

Da die IGeLU 2019 nicht in Europa stattfand, wurde mit Ex Libris am 24. September 2019 ein WebEx-Call anstelle des jährlichen Supportmeetings abgehalten. Besprochen wurde wie jedes Jahr unter anderem die Servicequalität des Supports, wofür von den Bibliotheken einige Beispiele zur Veranschaulichung vorgebracht wurden. Ein wichtiger Punkt war die Darstellung der Schlagwortketten im NUI: Schlagwortketten wurden nicht mehr in einer Zeile dargestellt, sondern die einzelnen Schlagwörter wurden untereinander angezeigt. Hier wurde von Ex Libris zugesagt, dass die einzeilige Darstellung wieder angeboten werden soll. Dies ist seit dem November Release 2019 auch bereits umgesetzt.

Außerdem wurde über den neuen Central Discovery Index, der den Primo Central Index ablöst, gesprochen. Hier wurde von Ex Libris präsentiert, welche Auswirkungen dieser neue Index hat und wie die Umstellung für den Verbund ablaufen wird. Außerdem wurde ein weiterer Call zu einem späteren Zeitpunkt vereinbart.

Im Bereich Statistik bzw. Auswertungen zur Suche spielt Primo Analytics eine wichtige Rolle. Allerdings gibt es hier von Ex Libris sehr wenig Dokumentation und die Trennung von Alma und Primo funktioniert nicht immer einwandfrei. Dennoch ist Primo Analytics die einzige Auswertungsmöglichkeit für die Bibliotheken, die schon auf das NUI umgestiegen sind.

\section{Primo-Teilnehmerversammlung}

Im Oktober 2019 fand die siebente Primo-Teilnehmerversammlung statt. Der Schwerpunkt lag auf dem Bericht der „Arbeitsgruppe Strategien und 
Perspektiven im Bereich Discovery im OBV“, die bei der letzten Versammlung einberufen wurde. Darüber hinaus wurden Berichte der Bibliotheken und des Primo-Beirats vorgestellt.

\subsubsection{Umstieg von Primo Central Index zu Central Discovery Index}

Für den Wechsel vom Primo Central Index $(\mathrm{PCI})$ auf den neuen Central Discovery Index (CDI) wurde im Zuge der Teilnehmerversammlung eine Implementierungsgruppe einberufen. Diese setzt sich aus Vertreterinnen und Vertretern von Primo und Teilnehmenden aus weiteren Bereichen zusammen. Die Implementierungsgruppe sammelt alle Informationen und leitet Fragen zur Umstellung an Ex Libris weiter.

Die Umstellung auf den CDI soll im Sommer 2020 erfolgen. Vorab erfolgt eine Testphase, in der beide Indizes parallel zu pflegen sind. In Primo ist jedoch bis zur definitiven Umstellung nur der PCl sichtbar.

\subsubsection{Primo-Wiki}

Mit Anfang Februar wurde die umfangreiche Umgestaltung und Aktualisierung des Primo-Bereichs im externen Wiki der OBVSG nach rund drei Monaten Arbeit abgeschlossen.

\subsubsection{Primo4Waves}

Im Berichtszeitraum sind Wave 5 und Wave 6 mit Alma in Produktion gegangen und wurden im Zuge dessen auf das NUI von Primo umgestellt. Die Technische Universität Graz ist zudem seit der Wave 6 neuer PrimoFlex-Kunde.

\subsubsection{Primo-Instanzen}

Stand der Primo-Instanzen mit März 2020:

\begin{tabular}{|l|c|c|}
\hline \multicolumn{1}{|c|}{ Primo-Instanz } & Institutionen & Bibliothekssystem \\
\hline prm40 & 21 & Aleph und Alma \\
\hline prm40-test & 2 & Aleph und Alma \\
\hline prm41 & 15 & Aleph \\
\hline prm42 & 15 & Alma \\
\hline prm42-test & 13 & Alma \\
\hline
\end{tabular}




\subsection{Bestandsdatenlieferung an die ZDB}

Die Neuimplementierung der ZDB-Bestandslieferung auf Basis von Alma ruht nach wie vor wegen permanenter Inanspruchnahme der erforderlichen Ressourcen durch andere, prioritäre Anforderungen.

\subsection{EasyTool}

EasyTool ist eine eigens für die Bedürfnisse des Bundesministeriums für Finanzen entwickelte Softwarelösung, die auf eine effiziente Exemplarverwaltung ausgelegt ist. Sie wird in diesem Rahmen laufend an die Bedürfnisse des Bundesministeriums für Finanzen adaptiert.

\subsection{MetaLib/SFX}

MetaLib ist ein Service zur gleichzeitigen Recherche in mehreren Datenbanken, SFX ein Auflösungsdienst für den Zugang zu elektronischen Ressourcen. Nachfolgende Einrichtungen nutzten im Berichtszeitraum diesen Dienst der OBVSG:

\begin{tabular}{|c|c|}
\hline System & Einrichtung \\
\hline SFX & Universität für angewandte Kunst Wien \\
\hline SFX & Parlament \\
\hline SFX & Universität für Musik und darstellende Kunst Wien \\
\hline SFX & FH Joanneum \\
\hline SFX & Universität Wien \\
\hline MetaLib & Veterinärmedizinische Universität Wien \\
\hline MetaLib & Wirtschaftsuniversität Wien \\
\hline MetaLib & Universität für angewandte Kunst Wien \\
\hline MetaLib & Universität Linz \\
\hline MetaLib &
\end{tabular}

Alma ersetzt SFX, dieses wird daher für umgestiegene Einrichtungen obsolet.

\subsection{E-Book-Datenversorgung}

Das monatliche Aufarbeiten der von Springer bereitgestellten Daten und deren Auslieferung an die am Dienst teilnehmenden Bibliotheken läuft zuverlässig und stabil. 
Nachdem die OBVSG nach dem Umstieg auf Alma ihren E-Book-Dienst evaluiert und anschließend überarbeitet hatte, stand im letzten Jahr die Analyse der Daten im Vordergrund. Auf Basis der in Österreich vorhandenen Lizenzen wurde begonnen, die im Springer Metadatastore angebotenen Titel mit denen im Verbundkatalog abzugleichen. Um Dubletten zu vermeiden, werden die im Verbundkatalog vorhandenen Titel vor dem Abgleich mit der DOI angereichert. Dadurch konnten bereits einige Lücken geschlossen werden.

\begin{tabular}{|l|c|}
\hline \multicolumn{1}{|c|}{ Springer } & $\begin{array}{c}\text { Neue Titel: aufbereitet und eingespielt } \\
\text { (Mai 2019-Februar 2020) }\end{array}$ \\
\hline Monatliche Lieferungen & 7.321 \\
\hline Neulizenzierung & 2.167 \\
\hline Lücken & 1.755 \\
\hline Summe & 11.243 \\
\hline
\end{tabular}

\begin{tabular}{|l|l|}
\hline Metadaten-Bearbeitung (Sigeltausch) & 2.778 \\
\hline DOI-Anreicherung & 8.173 \\
\hline
\end{tabular}

Es wurden folgende Aufbereitungen von externen E-Book-Metadaten durchgeführt:

- Oxford Scholarship Online Philosophy Collection

- Bloomsbury EBook-Pakete

- Verlag IGI Global 2017-19

- Ebsco eBooks Nursing Collection

- Mohr Siebeck

Aktuell werden für folgende Einrichtungen monatlich Springer-Daten verarbeitet, wobei für Aleph- und Alma-Bibliotheken verschiedene Verfahren anzuwenden sind:

- FH Oberösterreich (Aleph)

- FH Technikum Wien (Aleph)

- FH Salzburg

- Universität für Bodenkultur Wien

- Universität Graz

- Universität Innsbruck

- Universität Klagenfurt

- Universität Linz

- Medizinische Universität Wien

- Universität Salzburg 
- Technische Universität Wien

- Universität Wien

- Wirtschaftsuniversität Wien

Für das Aufbereiten externer Metadaten, welche in die jeweilige Alma-Bibliothek, geteilt mit der Netzwerkzone, eingespielt werden, ist weiterhin geplant ein Webinterface zu entwickeln.

Mit der zahlenmäßigen Zunahme der Alma-Bibliotheken mit jeder Wave hat sich die Nachfrage nach Wissen zum Umgang mit elektronischen Ressourcen erhöht. Durch die monatlichen Telefonkonferenzen der Arbeitsgruppe EG-E-Medien, die zweimal jährlich stattfindenden physischen Treffen, die rege benutzte Mailingliste sowie die von Teilnehmern dieser Arbeitsgruppe durchgeführten Workshops konnten gemeinsam erarbeitete Best-Practice-Empfehlungen einer breiten Anzahl von Personen zur Kenntnis gebracht werden. Neue Entwicklungen in Alma werden in der Arbeitsgruppe diskutiert und über die Mailingliste allen Alma-Teilnehmern bekannt gemacht.

\subsection{Bibliotheksstatistik}

Im Berichtszeitraum gab es keine besonderen Vorkommnisse. Der Dienst wird vom hbz normal bereitgestellt.

\subsection{Adressdatenbank (ACC09) / OBVSG als ISIL-Agentur}

Die OBVSG ist die offizielle Registration Agency für die österreichweite Vergabe von weltweit eindeutigen Bibliothekscodes (Bibliothekssigel). Das internationale System für Bibliothekssigel bildet der International Standard Identifier for Libraries and Related Organizations (ISIL).

Im Berichtszeitraum wurden 12 neue Informationseinrichtungen inklusive ISILs in die Datenbank aufgenommen und 44 bestehende bearbeitet.

Die als Basis dienende Adressdatenbank ACC09 läuft vorerst weiter in Aleph und muss spätestens bis zum Abschalten desselben durch eine andere technische Lösung ersetzt werden.

\subsection{Visual Library}

Die OBVSG bietet in Zusammenarbeit mit den Firmen semantics Kommunikationsmanagement und Walter Nagel mit Visual Library ein Repositorium als Konsortialmodell an. Die OBVSG sorgt für die nötige 
Infrastruktur (Hardware, Software, Datensicherung), übernimmt die erforderliche Systemadministration und verwaltet Bestellungen und Verrechnung. Eine Integration des Dienstes in bestehende Verbundworkflows und die Datenversorgung der Primo-Suchmaschine werden ebenfalls gewährleistet.

\subsubsection{Stand der Implementierung}

Der Visual Library Server wurde im Dezember 2019 auf die „Version 1906“ aktualisiert. Zahlreiche Verbesserungen, Neuimplementierungen und Fehlerkorrekturen wurden damit eingebracht. Zum Beispiel:

- Plan-S: Umsetzung in Visual Library

- Statistikmodul

- GND in Visual Library

- Modulare, individuell anpassbare Erfassungs- und Hochladebestätigungen

- Gänzliche Umstellung der Uploadformulare für Hochschulschriften und Open-Access-Artikel auf responsives Webdesign

\subsubsection{Teilnehmende}

Aktuell nehmen den Dienst produktiv in Anspruch (PS = Publikationsserver, ZS = Zeitschriftenserver, RD = Retrodigitalisierung):

\begin{tabular}{|l|c|c|c|}
\hline \multicolumn{1}{|c|}{ Einrichtung } & \multicolumn{2}{c|}{ Modul } \\
\hline $\begin{array}{l}\text { Internationale Stiftung Mozarteum / } \\
\text { Bibliotheca Mozartiana }\end{array}$ & & & RD \\
\hline FH BFI Wien & PS & & \\
\hline FH Campus Wien & PS & & \\
\hline FH Joanneum Graz & PS & & \\
\hline Medizinische Universität Wien & PS & & \\
\hline Technische Universität Wien & PS & ZS & \\
\hline Universität für Bodenkultur & PS & ZS & \\
\hline Universität Graz & PS & ZS & RD \\
\hline Universität Innsbruck & PS & ZS & RD \\
\hline Universität Klagenfurt & PS & ZS & \\
\hline Universität Linz & PS & & \\
\hline Universität Salzburg & PS & ZS & \\
\hline Universität Mozarteum Salzburg & PS & & \\
\hline
\end{tabular}




\subsubsection{Organisatorisches}

\section{Visual-Library-Workshop}

Am 2. Juli 2019 wurde an der OBVSG ein Workshop gemeinsam mit den Visual-Library-Anwenderinnen und -Anwendern abgehalten mit dem Ziel, die Workflows anzupassen, sowie neue Formulare für die Dokumentenabgabe zu definieren, bzw. bestehende zu adaptieren.

Die Ergebnisse wurden an Semantics kommuniziert und werden nach Möglichkeit in künftige Weiterentwicklungen einfließen.

\section{Visual Library-Anwendertreffen}

Am 24. September 2019 fand an der OBVSG das 5. Visual Library Anwendertreffen statt, erstmals mit Vertretern von Semantics und Walter Nagel.

Dabei wurden künftige funktionale Erweiterungen von Visual Library vorgestellt, sowie Erfahrungen und Best-Practices in der Anwendung von Visual Library besprochen.

\section{Telekonferenzen}

Mit Semantics findet im Sechs-Wochen-Rhythmus eine Videokonferenz statt, um aktuelle wie auch strategische Themen zu besprechen.

\subsubsection{Ausblick}

Für die Umstellung der Integration von Visual Library im OBV von MAB2 auf MARC21 wird ein Treffen mit Semantics stattfinden.

Das nächste Visual Library Update (Mitte des Jahres 2020) wird u.a. ein verbessertes Statistikmodul bringen.

\subsubsection{Statistikwerte}

Siehe Abschnitt 7.15.7.

\subsection{URN(-Resolver)}

Der URN-Resolver der OBVSG steht allen interessierten Personen und Institutionen in Österreich zur Verfügung. Die archivierten Netzpublikationen erhalten einen URN aus dem Namensraum „urn:nbn:at“. Diese werden 
insbesondere für Online-Hochschulschriften sowie für Netzpublikationen vergeben, die auf vertrauenswürdigen Dokumentenservern verwaltet werden. Das Zitieren einer URN ist ein sicherer Weg langfristig auf ein digitales Objekt zu verweisen.

Folgende Institutionen bzw. österreichische URN-Namensräume sind im Berichtszeitraum hinzugekommen:

- Medizinische Universität Wien | OA-Publikationen

- Universität Wien / Bibliotheks- und Archivwesen | Goobi-Publikationen

Ein Überblick aller aktuell aktiven Namensräume, die am österreichischen URN-Resolver registriert sind, findet sich unter https://resolver.obvsg.at/ namespaces.

\subsection{Memo-Workflow}

Siehe Abschnitt 3.4.1.

\subsection{Statistikwerte aus den OBVSG-Diensten}

\subsubsection{IVSCAN - Aufsatzdaten der ÖNB}

Für die Primo-Einrichtungen werden im regulären Betrieb die IVSCANDaten der Monographien in die entsprechenden Sichten übertragen. Mit Stand 1. März 2020 ergibt sich folgende Nutzung:

\begin{tabular}{|l|c|c|c|}
\hline \multicolumn{1}{|c|}{ Institution } & 2020 & 2019 & 2018 \\
\hline Universität Wien & 795.064 & 779.584 & 750.584 \\
\hline Universität Salzburg & 516.351 & 506.160 & 485.340 \\
\hline Universität Graz & 493.179 & 483.954 & 466.299 \\
\hline Universität Innsbruck & 482.065 & 474.339 & 451.788 \\
\hline Universität Klagenfurt & 251.285 & 248.180 & 236.452 \\
\hline Oberösterreichische Landeskultureinrichtungen & 125.605 & 209.597 & 209.597 \\
\hline Universität Linz & 167.568 & 164.766 & 154.424 \\
\hline Wirtschaftsuniversität Wien & 145.227 & 142.707 & 131.928 \\
\hline Technische Universität Wien & 0 & 111.814 & 111.814 \\
\hline Verbund für Bildung und Kultur & 89.438 & 88.143 & 82.250 \\
\hline Parlamentsbibliothek & 93.964 & 54.775 & 54.775 \\
\hline Universität für angewandte Kunst Wien & 60.959 & 61.166 & 52.734 \\
\hline Universität für Musik und darstellende Kunst Wien & 53.998 & 49.311 & 49.311 \\
\hline Medizinische Universität Wien & 43.716 & 43.388 & 42.736 \\
\hline
\end{tabular}




\begin{tabular}{|l|c|c|c|}
\hline \multicolumn{1}{|c|}{ Institution } & 2020 & 2019 & 2018 \\
\hline Veterinärmedizinische Universität Wien & 11.675 & 11.520 & 11.174 \\
\hline Fachhochschule Sankt Pölten & 23.148 & 22.736 & 20.876 \\
\hline Fachhochschule Oberösterreich & 18.645 & 18.214 & 18.214 \\
\hline Bundesministerium für Finanzen & 22.476 & 22.323 & - \\
\hline Wienbibliothek & 124.305 & - & - \\
\hline Technische Universität Graz & 124.290 & - & - \\
\hline Fachhochschule Joanneum & 19.619 & - & - \\
\hline
\end{tabular}

\subsubsection{Automatische Identifikation und Verarbeitung von DNB PDF-TOCs}

Mit Stand 5. Februar 2020 befinden sich 251.752 DNB-Inhaltsverzeichnisse in eDOC.

\subsubsection{Schnittstelle DigiTool - Verbundkatalog}

Mit Stand 20. Februar 2020 befinden sich an 26.763 Verbundtiteln Links zur DigiTool-Anwendung an der Österreichischen Nationalbibliothek.

\subsubsection{ABO-Links in Verbundtiteln}

Mit Stand 20. Februar 2020 sind 454.207 ABO-Volltextlinks in den jeweiligen Verbundtitel eingebracht.

\subsubsection{EDOC-Objekte}

Die Gesamtzahl der Objekte im eDOC-Repository beträgt mit Stand 5. Februar 2020 1.157.606 Objekte, davon:

\begin{tabular}{|l|c|c|}
\hline & 2020 & 2019 \\
\hline Inhaltsverzeichnisse & 768.990 & 737.791 \\
\hline Abstracts & 45.697 & 45.400 \\
\hline Volltexte & 62.433 & 54.083 \\
\hline
\end{tabular}

\subsubsection{URN-Statistik}

Mit Stand Februar 2020 sind insgesamt 166.664 URN-Einträge registriert. Die Anzahl der URNs pro Institution finden Sie in nachfolgenden Tabelle: 


\begin{tabular}{|l|c|}
\hline \multicolumn{1}{|c|}{ Einrichtung } & Anzahl \\
\hline Universität Wien & 50.556 \\
\hline Compass-Verlag GmbH & 28.504 \\
\hline Österreichische Nationalbibliothek & 20.764 \\
\hline Universität Graz & 20.242 \\
\hline Technische Universität Wien & 14.479 \\
\hline Universität Innsbruck & 5.193 \\
\hline Internationale Stiftung Mozarteum / & 4.379 \\
\hline Bibliotheca Mozartiana & \\
\hline Arbeiterkammer Wien & 3.889 \\
\hline Universität Linz & 3.426 \\
\hline Universität Klagenfurt & 3.330 \\
\hline Medizinische Universität Wien & 3.130 \\
\hline Oberösterreichische Landesbibliothek & 2.876 \\
\hline Universität für Bodenkultur & 2.610 \\
\hline Universität Salzburg & 2.057 \\
\hline Fachhochschule Campus Wien & 816 \\
\hline Fachhochschule des BFI Wien & 214 \\
\hline Universität für Musik und darstellende Kunst Wien & 101 \\
\hline Universität Mozarteum Salzburg & 60 \\
\hline Fachhochschule Joanneum & 29 \\
\hline
\end{tabular}

\subsubsection{Visual Library}

Mit Stand Februar 2020 werden an der OBVSG 14 Einrichtungen mit 144 Subdomains vorgehalten:

- 120.680 Katalogisate, [+51,23\%]

- 262.470 Strukturdaten [+24,60\%] und

- 2.842.980 Digitalisate (Seiten) [+34,38\%] 
Im VL-Portal sind mit Stand Februar 2020 nachgewiesen:

\begin{tabular}{|c|c|c|c|c|c|c|c|c|c|c|c|c|c|}
\hline 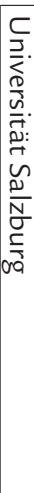 & 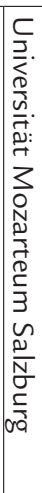 & 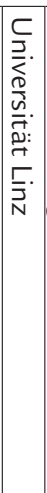 & 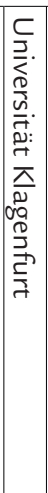 & 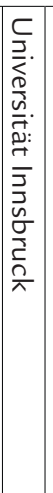 & 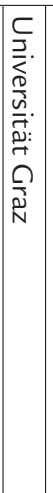 & 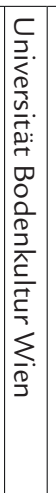 & 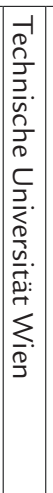 & 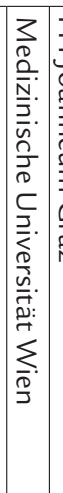 & 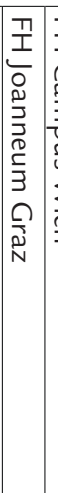 & 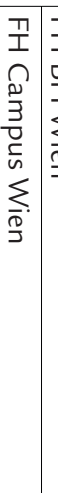 & 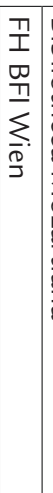 & 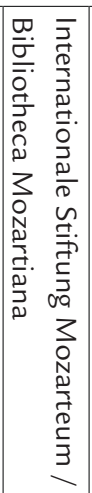 & 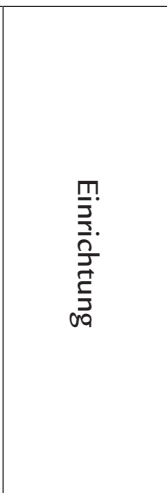 \\
\hline $\begin{array}{l}\vec{\infty} \\
N\end{array}$ & $a$ & $\begin{array}{l}\omega \\
\dot{N} \\
v \\
v\end{array}$ & $\begin{array}{l}\omega \\
\overrightarrow{\vec{v}} \\
\sigma\end{array}$ & $\begin{array}{l}9 \\
0 \\
\infty \\
0 \\
0\end{array}$ & \begin{tabular}{|l}
$\vec{\Delta}$ \\
$\dot{u}$ \\
$\overrightarrow{\vec{V}}$
\end{tabular} & $\begin{array}{l}\sim \\
\omega \\
\Omega \\
-\end{array}$ & $\begin{array}{l}\vec{\omega} \\
\dot{\Delta} \\
\omega \\
\omega\end{array}$ & $\begin{array}{l}\omega \\
\omega \\
\infty \\
\rightarrow\end{array}$ & $\overrightarrow{6}$ & $\begin{array}{l}\overrightarrow{\vec{A}} \\
\dot{\sim} \\
\tilde{H} \\
\vec{A}\end{array}$ & N & & 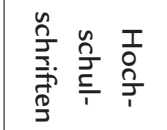 \\
\hline & & & & $\begin{array}{l}\omega \\
\omega \\
\omega \\
\omega \\
N\end{array}$ & $\begin{array}{l}N \\
a \\
\stackrel{+}{v}\end{array}$ & & & & & & & $\begin{array}{l}\omega \\
\dot{\omega} \\
\vec{D}\end{array}$ & 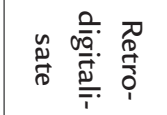 \\
\hline$\vec{v}$ & શิ & $\stackrel{N}{D}$ & $\vec{\Xi}$ & ğ & $\begin{array}{l}\infty \\
0 \\
-\end{array} \mid$ & \begin{tabular}{|l}
$\omega$ \\
$\omega$ \\
$\omega$
\end{tabular} \mid & $\stackrel{0}{\circ}$ & $\begin{array}{l}\overrightarrow{\vec{v}} \\
\dot{\circ}\end{array}$ & & 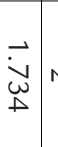 & $N$ & & 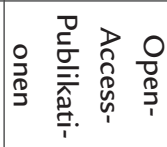 \\
\hline$\frac{\omega}{\vec{v}}$ & & & $\begin{array}{l}N \\
\Omega \\
\Omega\end{array}$ & $\begin{array}{l}\overrightarrow{\vec{v}} \\
\overrightarrow{\vec{v}}\end{array}$ & $\begin{array}{l}\omega \\
\dot{\omega} \\
\omega \\
v\end{array}$ & $\begin{array}{l}\omega \\
\omega \\
\infty\end{array}$ & $\frac{\Delta}{\omega}$ & & & & & & 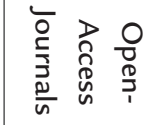 \\
\hline 6 & $V$ & $N$ & $\infty$ & $\vec{\infty}$ & 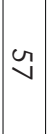 & $V$ & $V$ & $N$ & $N$ & $\Delta$ & $N$ & $\Delta$ & 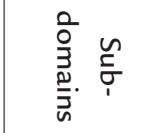 \\
\hline$\vec{v}$ & $\omega$ & जु & $N$ & $\begin{array}{l}+ \\
\dot{0} \\
\stackrel{2}{\omega}\end{array}$ & $\begin{array}{l}\grave{\partial} \\
\omega\end{array}$ & $\begin{array}{l}\infty \\
+ \\
\end{array}$ & $\tilde{\omega}$ & $\vec{v}$ & & & & $\begin{array}{l}u \\
\dot{\omega} \\
\stackrel{\Delta}{\Delta}\end{array}$ & 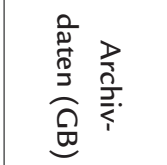 \\
\hline $\begin{array}{l}N \\
N \\
\text { un } \\
\alpha\end{array}$ & ত & $\begin{array}{l}\omega \\
u \\
\sigma \\
\sigma\end{array}$ & $\begin{array}{l}\omega \\
\vec{N} \\
N\end{array}$ & \begin{tabular}{l}
$\mathbb{D}$ \\
$\stackrel{0}{0}$ \\
$\infty$ \\
\hdashline
\end{tabular} & $\begin{array}{l}n \\
\dot{a} \\
\dot{\sigma} \\
u \\
c\end{array}$ & $\begin{array}{c}\omega \\
\dot{\omega} \\
\tilde{N}\end{array}$ & $\begin{array}{l}\vec{\Delta} \\
\dot{\omega} \\
\vec{v}\end{array}$ & $\begin{array}{l}\vec{p} \\
\dot{\infty} \\
\infty \\
\infty\end{array}$ & $\vec{b}$ & $\begin{array}{l}\vec{u} \\
i \\
\infty \\
\infty \\
\infty\end{array}$ & $\begin{array}{l}N \\
\Omega \\
-\end{array}$ & $\begin{array}{l}\dot{\omega} \\
\dot{\omega} \\
\vec{D}\end{array}$ & 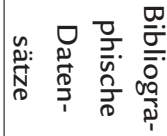 \\
\hline Y & $\stackrel{\vec{\infty}}{\vec{\Delta}}$ & $\begin{array}{l}\omega \\
\stackrel{D}{+} \\
\stackrel{\vec{D}}{\circ} \\
\stackrel{N}{N}\end{array}$ & $\begin{array}{l}\stackrel{\vec{\Delta}}{N} \\
\stackrel{N}{N}\end{array}$ & $\begin{array}{l}\overrightarrow{\vec{n}} \\
\overrightarrow{\vec{\Delta}} \\
\dot{\alpha} \\
\vec{\Delta}\end{array}$ & $\begin{array}{l}\vec{N} \\
N \\
\vec{N} \\
0\end{array}$ & $\begin{array}{l}w \\
\stackrel{N}{N} \\
\\
V \\
V\end{array}$ & $\mid \begin{array}{l}n \\
6 \\
i n \\
u \\
-1\end{array}$ & $\begin{array}{l}N \\
\omega \\
0 \\
. \\
\overrightarrow{0}\end{array}$ & & & & $\begin{array}{l}\vec{a} \\
\stackrel{y}{0} \\
\overrightarrow{0}\end{array}$ & $\begin{array}{l}\frac{n}{D} . \\
\frac{\Phi}{\frac{D}{J}}\end{array}$ \\
\hline
\end{tabular}




\section{Termine}

Aufgrund der Fülle an Terminen ist die folgende Aufstellung nur eine Auswahl ohne Anspruch auf Vollständigkeit:

- Visual Library-Kundengespräch über Verbesserungserfordernisse am 17. Mai 2019 an der OBVSG

- Besprechung mit einer Delegation aus NRW zu Alma am 28. Mai 2019 an der OBVSG

- ZR/LR-SE-Sitzung am 12. Juni 2019 in Wien

- DACHELA von 25. Juni bis 26. Juni 2019 in Mannheim

- Visual Library-Workshop am 2. Juli 2019 an der OBVSG

- ZR-FE-Sitzung am 3. Juli 2019 an der OBVSG

- Standardisierungsausschuss am 4. Juli 2019 in Frankfurt am Main

- 11. Vollversammlung der Vertragsperiode 2018-2020 der Kooperation E-Medien Österreich am 4. Juli 2019 in Linz

- Alma-Schulung Wave 5 von 22. bis 26. und von 29. bis 30. Juli 2019 an der OBVSG

- IGELU 2019 und Developer's Day von 26. bis 29. August 2019 in Singapur

- GND-Redaktionstreffen am 9. September 2019 in Graz

- Österreichischer Bibliothekartag von 10. bis 13. September 2019 in Graz

- EISZ-Consortium-Meeting Budapest mit Focus on Open Science, 16. bis 17. September 2019

- 12. Vollversammlung der Vertragsperiode 2018-2020 der Kooperation E-Medien Österreich am 19. September 2019 an der OBVSG

- Visual Library-Anwendertreffen am 24. September 2019 an der OBVSG

- Open-Access-Tage von 30. September bis 2. Oktober 2019 in Hannover

- ZR-FE-Sitzung am 2. Oktober 2019 an der OBVSG

- ZR-Sitzung am 9. Oktober 2019 an der OBVSG

- 71. Frankfurter Buchmesse 16. bis 18. Oktober 2019

- ICOLC-Meeting Luxemburg 21. bis 23. Oktober 2019

- 7. Versammlung der Primo-TeilnehmerInnen am 29. Oktober 2019 an der OBVSG

- GND-Multiplikatorinnenschulung am 6., 12. und 19. November 2019 an der OBVSG

- 13. Vollversammlung der Vertragsperiode 2018-2020 der Kooperation E-Medien Österreich am 7. November 2019 an der OBVSG 
- 37. Treffen der Systembibliothekarinnen und Systembibliothekare von 13. bis 14. November 2019 an der AK Bibliothek für Sozialwissenschaften in Wien

- AG Verbundsysteme am 19. und 20. November 2019 in Köln

- Jubiläumsfeier 20 Jahre Oberösterreichische Landesbibliothek am 23. November 2019 in Linz

- Alma-Schulung Wave 6 von 25. bis 28. November 2019 an der OBVSG

- ZR-FE-Sitzung am 12. Dezember 2019 an der OBVSG

- Standardisierungsausschuss am 12. Dezember 2019 in Frankfurt am Main

- Verabschiedung von GDin Dr. ${ }^{\text {in }}$ Elisabeth Niggemann sowie Amtseinführung von GD Frank Scholze am 13. Dezember 2019 in Frankfurt am Main

- 14. Vollversammlung der Vertragsperiode 2018-2020 der Kooperation E-Medien Österreich am 19. Dezember 2019 an der OBVSG

- GASCO-Treffen Frankfurt 24. Jänner 2020

- 15. Vollversammlung der Vertragsperiode 2018-2020 der Kooperation E-Medien Österreich am 30. Jänner 2020 an der OBVSG

- Digitaler Assistent-Multiplikatorenschulung von 18. bis 19. Februar 2019 an der OBVSG

- GND-in-Alma-Schulung Wave 6 am 25. Februar 2020 an der OBVSG

- Sharingtag und Treffen der Alma-ASP-Bibliotheken am 27. Februar 2020 an der OBVSG

- GND-Redaktionstreffen am 10. März 2020 an der OBVSG

- Übergang zum COVID-19-Notbetrieb am 16. März 2020

- ZR-FE-Sitzung am 25. März 2020 über Telekonferenz

- AG Verbundsysteme am 29. April 2020 über Telekonferenz

\section{Organisatorisches und Sonstiges}

\subsection{IGeLU}

Die OBVSG engagiert sich weiterhin in den Bereichen Softwareweiterentwicklung und strategische Planung für die eingesetzten Produkte. Dazu gehört insbesondere eine aktive Mitarbeit in der International Group of Ex Libris Users (IGeLU), der über 350 Institutionen aus über 40 Ländern aller fünf Kontinente angehören. 
Die OBVSG besetzt fünf unterschiedliche Funktionen bei der IGeLU:

- Member Primo Product Working Group

- Module Coordinator for Aleph Enhancements

- Member Alma Product Working Group

- Member Alma Authority Focus Group

- Member SWIG Consortia

\subsection{Geplante Novellierung OBVSG-Gesetz}

Das Bundesgesetz über die Errichtung der OBVSG ist am 1. Jänner 2002 in Kraft getreten. In der Zwischenzeit haben sich viele Gebiete weiterentwickelt und neue sind dazugekommen. Daher wird schon seit einiger Zeit an eine Aktualisierung des Gesetzes gedacht. Vorgesehen ist unter anderem die Übernahme der Verbundzentrale des Verbundes für Bildung und Kultur und eine Zusatzdotation für umschriebene neue Dienste (allerdings weiterhin keine Erhöhung der seit Anbeginn gleichgebliebenen Basisabgeltung des Bundes) sowie die Einrichtung eines Beirats aus Vertretungen der Verbundbibliotheken. Auf Grund der Entwicklungen nach dem sogenannten „Ibiza-Video“ konnte die Novelle 2019 nicht mehr in den Gesetzgebungsprozess eingespeist werden. Erst nach den Neuwahlen und Bildung der Regierung, aber noch vor Verhängung der COVID-19-Pandemiemaßnahmen wurde der Referentenentwurf zur Begutachtung ausgesandt. Die Frist dazu ist am 2. April 2020 abgelaufen und es bestehen nun realistische Chancen, dass die Novelle im Parlament behandelt und zum 1. Jänner 2021 in Kraft tritt - ein Jahr später als ursprünglich beabsichtigt. ${ }^{3}$

\section{Österreichische Bibliothekenverbund und Service GmbH (OBVSG) E-Mail: office@obvsg.at}

1 https://ted.europa.eu/udl?uri=TED:NOTICE:454959-2019:TEXT:DE:HTML

2 Als Nebeneffekt sind damit auch ausreichend viele Notfallgeräte für die Heimarbeit während der COVID-19-Beschränkungen verfügbar.

3 Nachtrag: Die Novelle zum OBVSG-Gesetz wurde am 24. Juli 2020 im Bundesgesetzblatt kundgemacht: https://www.ris.bka.gv.at/Dokumente/BgblAuth/BGBLA_2020_I_80/BGBLA_2020_I_80.html 